\title{
Teaching Materials to Provoke Students' Study Interests from the Experimental Phenomena of Electrospinning
}

\author{
Xiao-Hong Chen*, Hua-Ling Fang, Zi-Yun Gong, Jun-Ming Xiang, Dengguang Yu \\ School of Materials Science and Engineering, University of Shanghai for Science and Technology, \\ 516 Jungong Road, Shanghai 200093, China. \\ Email: Cxh992@163.com \\ *Corresponding author
}

\begin{abstract}
After many years of hard studies in classroom, the college students are often tired of the boring learning of theoretical knowledge. How to draw their enough attention to theories of professional knowledge poses a big threat to their teachers. This paper develops a learn procedure for the college students to broaden the level of knowledge and to deepen their professional knowledge. Electrospinning experimental phenomena are explored as the teaching materials to provoke students' interests of studying related electrohydrodynamic theories. The results demonstrated that that the feedback teaching of scientific research can be more effective in imparting professional knowledge to undergraduate students. This paper shows an innovative way of refining teaching materials from advanced nanotechnologies for higher education.
\end{abstract}

Keywords-Teaching materials; Experimental phenomenon; Electrospinning; Higher education

\section{INTRODUCTION}

Everyday, we can see numerous things happened before our eyes. Our ancestors got to know the world just through observing, recording and analyzing the natural phenomena that happened around them. Many life experiences have been passed down from generation to generation and some unexplained phenomena become superstitious. The modern industrial revolution led to the rapid expansion of human knowledge of the world. However, these knowledge is mainly about the natural eye-able world and can be very useful to explain the eye-able phenomena. One typical example is the Newton's law of universal gravitation (Fig.1). The story is familiar to everyone that the apple hit Newton's head and led to the discovery of gravity.

Today we are in a nanoscale Era. It is impossible that we achieve new knowledge directly through our seeing, listening, touching and sensing. The phenomena happened at microscale and nanoscale must be magnified before they can be perceptual It is just this strong willing, a large bodies of modern instruments have been developed for us to explore the micro world. These instruments include optical microscope, scanning electron microscope, transmission electron microscope, atomic force microscope, X-ray diffraction and so on. With these powerful tools, many new and unexpected phenomena can be captured easily, which in turn will provide hints for us to get new knowledge about the material world. Certainly, these phenomena should be excellent teaching materials for imparting cutting-edge knowledge to the undergraduate students.

In this paper, with two experimental phenomena happened around electrospinning (an advanced nanotechnology for creating polymeric nanofibers) as teaching materials, a new approach about how to provoke the college students' interests about theoretical learns is exhibited in details. One phenomenon is observed using the optical microscope and is about the electrospinning process. The other phenomenon is recorded using a scanning electron microscope and is about the electrospun nanoproducts.

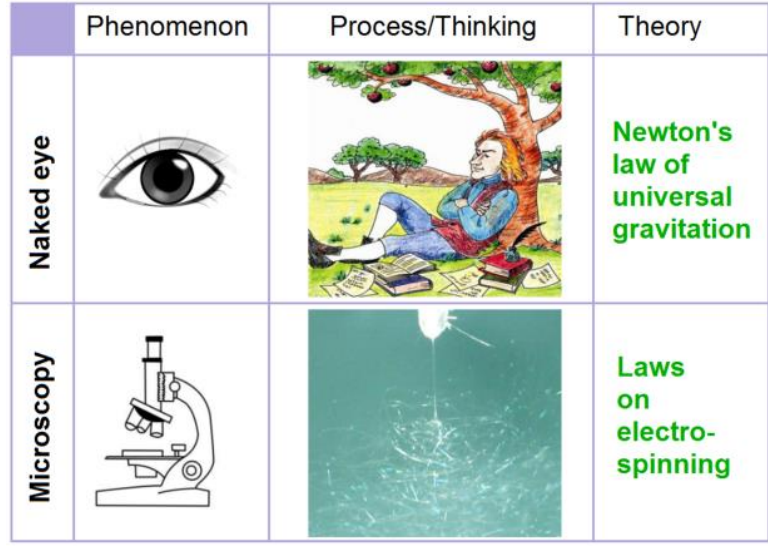

Fig. 1 A schematic diagram showing the achievement of knowledge from phenomena. 


\section{ELECTROSTATIC SPINNING}

Spinning is an industrial process that is exploited to create fibers from polymer. After many years' development, a series of spinning technologies have been developed such as wet spinning, dry spinning, dry-jet-wet spinning, melt spinning, gel spinning, and also electrospinning. For implementing a spinning process, the polymer being spun must be converted into a liquid state (melted or dissolved in a solvent). The liquid is forced through a spinneret (with it to be cooled or removing of the solvent) to achieve the solidified filament products.

In textile field, wet spinning is the oldest and most common processes. This process is used for polymers that need to be dissolved in a solvent to be spun. The spinneret is submerged in a chemical bath that causes the fiber to precipitate, and then solidify. The process gets its name from this "wet" bath. Compared with the wet spinning process, electrostatic spinning (now is frequently called electrospinning) also starts from the polymer solution or melt. But electrospinning exploits an electrical charge to draw very fine fibers (typically on the micro or nano scale). It thus shares characteristics of both electrospraying and conventional solution dry spinning of fibers [1-5]. The process does not require the use of coagulation chemistry or high temperatures to produce solid threads from solution. This makes the process particularly suited to the production of fibers using large and complex molecules. .

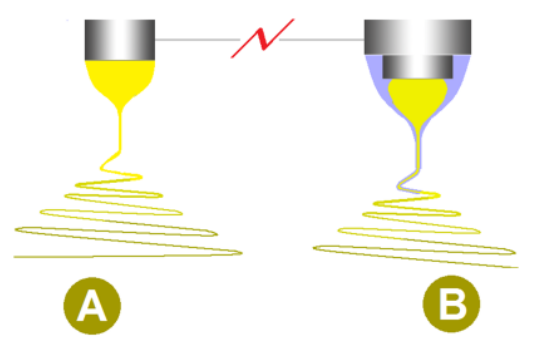

Fig. 2 A diagram showing the working processes of blending electrospinning (A) and coaxial electrospinning (B).

During the past two decades, electrospinning is developing very fast toward three directions. One is the simultaneous treatments of multiple working fluids, which lead to the generations of coaxial electrospinning, modified coaxial electrospinning, tri-axial electrospinning, side-by-side electrospinning and their combinations [6-11]. The second is the creation of electrospun polymeric nanofibers on a large scale [12-13]. And the third is its integration with other technologies (e.g. electrospraying) for developing new electrospinning-based nanotechnologies [14-15]. However, the main stream in this region is still about the most common single-fluid blending electrospinning and the double-fluid coaxial electrospinning (Fig.2). By estimation, the publications about these two electrospinning processes occupy over $99 \%$ of the whole related articles.

\section{ABNORMAL AND NORMAL PHENOMENA DURING ELECTROSPINNING PROCESSES}

Typically, an electrospinning working process consists of five successive steps, i.e. charge of the working fluid, formation of a Taylor cone, a straight fluid emitted from the Taylor cone, an unstable region full of bending and whipping, and collection of the final solid nanofibers. These steps can not be observed directly through naked eyes because of the applied voltage and small size of the working microfluids. However, the interesting phenomena during the processes can be viewed under a suitable magnification. Shown in Fig. 3A is an abnormal phenomenon about electrospinning of zein solution [16]. It is clear that the yellow semi-solid substance gradually accumulated and hanged on the nozzle of spinneret until the electrospinning process was totally stopped. Although these semi-solid substance can be removed manually and the electropsun nanofibers can go on to deposit on the fibercollected electrode, the working process is abnormal. A normal electrospinning process should be a continuous and robust process with smooth preparation and deposition of nanofibers.

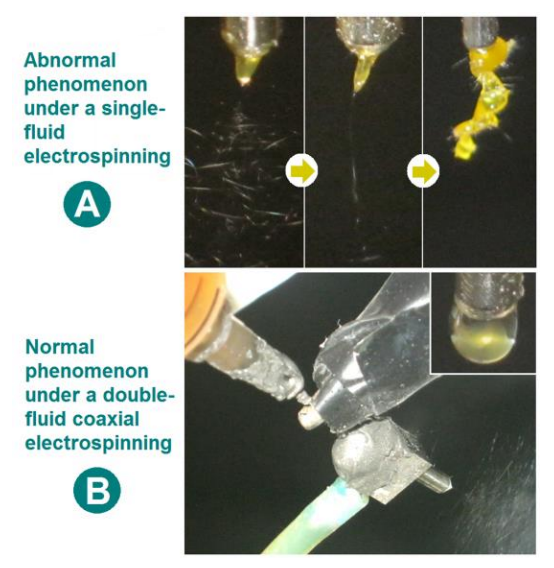

Fig. 3 An abnormal phenomenon of a single-fluid blending electrospinning (A) and a normal phenomenon of a double-fluid modified coaxial electrospinning (B).

However, when a pure solvent was exploited as a sheath working fluid to surround the core zein working solution, the electrospinning process became a normal working process, as shown in Fig. 3B. The upper-right inset shows a typical compound core-shell droplet with the transparent sheath solvent surrounding the yellow zein solution. After a suitable high voltage was applied, the coaxial electrospinning processes were carried out continuously and robustly without any manual interventions. These phenomena greatly provoke the students' interests about this advanced nanotechnology. The systematic explanations on these normal and abnormal phenomena not only make the students grasp the meaning of electrohydrodynamic atomization better and deepen their professional knowledge, but also promote them to strengthen practice training and think of other similar technology such as electrospraying [17-23]. 


\section{ABNORMAL AND NORMAL PHENOMENA ABOUT THE ELECTROSPUN NANOPRODUCTS}

In the traditional sense, the electrospun nanofibers should have a cylindric morphology. However, the created nanofibers using zein solutions as working fluids are "abnormal". Shown in Fig. 4, no matter nanofibers prepared from the single-fluid blending electrospinning or nanofibers fabricated from the double-fluid coaxial electrospinning, they all show a nanoribbon morphology, as indicated by their scannning electron microscopic images. These apparent "abnormal" phenomena are truly completely normal phenomena. The understanding of this interesting point would broaden their knowledge about material science and engineering.

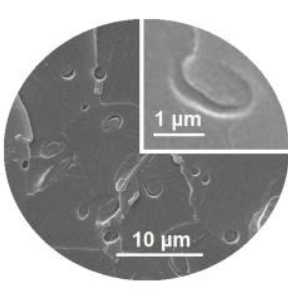

A

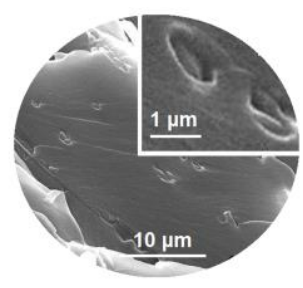

B
Fig. 4 The scanning electron microscopic images of the electrospun nanofibers from the blending electrospinning (A) and coaxial electrospinning (B).

\section{THE PHENOMENA ARE EXCELLENT START POINTS FOR EXPANDING THEIR PROFESSIONAL VISION AND FOR DEEPENING THEIR THEORETICAL KNOWLEDGE}

The phenomena mentioned above, regardless of normal or abnormal, should be excellent start points of teaching materials for expanding the students' professional vision and also for deepening their theoretical knowledge. Shown in Fig. 5, the phenomena of the electrospinning processes should give a very deep impression on the students. With these phenomena as the starting points, many new knowledge about electrospinning should be easier to be understood. And then, the peer of electrospinning, i.e. electrospraying can be imparted to the students in a comparative manner because both electrospinning and electrospraying belong to the electrohydrodynamic atomization processes [24-26]. Certainly, more knowledge about materials science and engineering can be further expanded for teaching.

The theroretical knowledge is always tedious for the students. However, when these tedious theories are associated with the interesting phenomena directly, the explanations should be more effective and easier for the students to grasp the key contents. Shown in Fig. 6, phenomena can be an excellent starting point for step-by-step upward learning. For example, the abnormal scanning electron microscopic images in Fig. 4 can be explained with their electrospinning processes. Their microformation mechanisms have a close relationship with the properties of zein solutions and also the operational parameters. Zein, as a plant protein, has a certain stereoscopic conformation of its molecules. The zein molecules also have strong secondary interactions such as hydrogen bondings and electrostatic interactions. In the electrospinning and drying processes, when the solvent in the working fluid evaporates quickly, a semi-solid substance would be precipitated. And meanwhile, because of strong interaction between the zein molecules with the metal nozzle, these semi-solid substance would cling and hang on the spinneret, creating the phenomenon in Fig. 3A. It is also because the easily formation of semi-solid substance on the surface of the working fluids, which would prevent the further evaporation of the inner solvent in the fluid jet. After these "outer-dried-inner-wet" jets or fibers are deposited on the collector, the inner trapped solvent would still penetrate into the atmosphere, which make the fibers be flattened by the atmospheric pressure, resulting morphology exhibited in Fig. 4A.

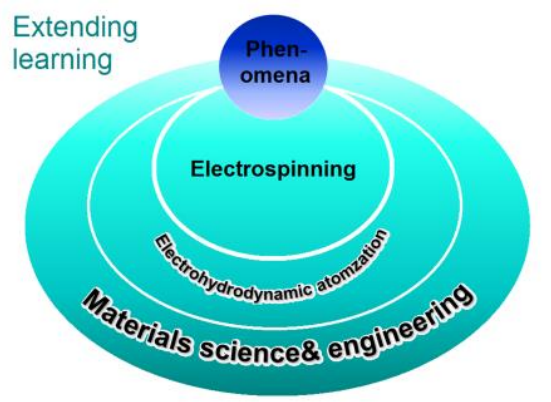

Fig. 5 Expansion of students' professional visions - a gradual expanding procedure for the college students to start from the experimental phenomena to the advanced technology, to the analogical techniques, and finally to their common knowledge in their discipline of materials science and engineering.

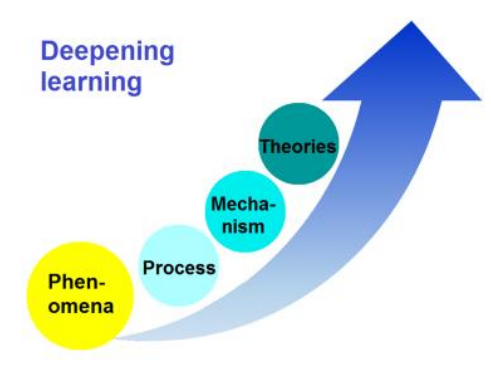

Fig. 6 Deepening the theoretical knowledge - a step-by-step upward manner for the college students to start from the experimental phenomena to the material treating process, to the fundamental mechanism, and finally to make clear about the related tedious theories.

When a pure solvent is exploited to smooth the electrospinning process, the influence of the sheath solvent should be profound [27-28]. The theory involved is Knudsen layer theory. Knudsen layer is also termed as evaporation layer. It is a thin layer of vapor near a liquid or solid, which is named after Danish physicist Martin Knudsen. The Knudsen layer thickness $\left(L_{\mathrm{c}}\right)$ can be estimated according to the following equation [29]:

$$
L_{\mathrm{c}}=\frac{k T_{s}}{\pi d^{2} p_{s}}
$$

where $p_{\mathrm{s}}$ is the saturated pressure, $T_{\mathrm{s}}$ is the temperature, $d$ is the solvent molecular diameter, and $k$ is the Boltzmann's 
constant. The intentional addition of a surrounding sheath solvent would effectively prevent the formation of semi-solid substance around the spinneret' nozzle for a smooth and continuous working process in Fig. 3B. This make the Knudsen layer play its role in the formation of zein nanofibers. However, these created fibers (shown in Fig. 4B) are even smaller and flatter. This is because the sheath solvent would increase the interactions of zein molecules, particularly the hydrogen bondings.

\section{SUMMARY}

There are numerous phenomena in thescientific researches. These phenomena can be useful teaching materials for college students to provoke their study interests on both advanced technologies and also the related theories. With several abnormal experimental phenomena during electrospinning and also the electrospun nanoproducts as the starting points of vivid teaching materials, a new approach about how to expanding the students' professional vision and how to deepen their theoretical knowledge is proposed. This demonstration provides an excellent example for the teaching with scientific research back feeding, which should be more efficacious in imparting professional knowledge to the students in higher education.

\section{ACKNOWLEDGMENT}

The financial supports from the following projects are appreciated: the Shanghai Education Science Research Project (C17058), the National Natural Science Foundation of China (No. 51373101), the 2017 Graduate curriculum reform project in USST, and the College Student Innovation Project of USST (Nos.SH20181).

\section{REFERENCES}

[1] Wang, X.K. Liu, X.H. Chen, D.G. Yu, Y.Y. Yang, and P. Liu, "Electrospun hydrophilic Janus nanocomposites for the rapid onset of therapeutic action of helicid," ACS Appl. Mater. Interfaces, vol.10, pp.2859-2867, 2018.

[2] Z. Zhang, W. Li, G. Wang, Y.L. Qu, and D.G. Yu, "Electrospun 4th generation solid dispersions of poorly water-soluble drug utilizing two different processes," J. Nanomater. Vol.2018, Article ID 2012140, 2018.

[3] Q. Wang, D.G. Yu, S.Y. Zhou, C. Li, and M. Zhao, "Fabrication of amorphous electrospun medicated-nanocomposites using a Teflonbased concentric spinneret," e-Polymer, vol.18, pp.3-11, 2018

[4] Y.H. Wu, D.G. Yu, J.J. Li, Q. Wang, H.P. Li, and X.Y. Li, "Medicated multiple-component polymeric nanocomposites fabricated using electrospraying," Polym. Polym. Compos., vol.25, pp.57-62, 2017.

[5] Q. Wang, D.G. Yu, L.L. Zhang, X.K. Liu, Y.C. Deng, and M. Zhao, "Electrospun hypromellose-based hydrophilic composites for rapid dissolution of poorly water-soluble drug," Carbohydr. Polym., vol.174, pp.617-625, Oct, 2017.

[6] D.G. Yu, C. Yang, M. Jin, G.R. Williams, H. Zou, X. Wang, et al, "Medicated Janus fibers fabricated using a Teflon-coated side-by-side spinneret," Colloid. Surface B, vol.138, pp.110-116, Feburary 2016.

[7] Y.H. Wu, D.G. Yu, H.P. Li, X.Y. Wu, and X.Y. Li, "Medicated structural PVP/PEG composites fabricated using coaxial electrospinning," e-Polymers, vol.17, pp.39-44, January 2017.

[8] J. Li, Chen Yang, Hai-Peng Li, Qing Wang, and Deng-Guang Yu, "Oral controlled release in accordance with drug adsorption biological rhythm provided by an electrospun structural amorphous solid dispersion,” J. Control. Release, vol.259, pp.e61-e62, 2017.

[9] D.G. Yu, H.P. Li, C. Yang, J.J. Li, Q. Wang, and G.R. Williams, "Double-pulsatile release core-shell fibers fabricated using modified tri-axial electrospinning,” J. Control. Release, vol.259, pp.e24-e25, 2017.

[10] C. Yang, D.G. Yu, D. Pan, X.K. Liu, X. Wang, S.W.A. Bligh, et al, "Electrospun pH-sensitive core-shell polymer nanocomposites fabricated using a tri-axial processes," Acta Biomater., vol.35, pp.77-86, April 2016.

[11] X. Liu, W. Shao, M. Luo, J. Bian, D.G. Yu, "Electrospun blank nanocoating for improved sustained release profiles from medicated gliadin nanofibers," Nanomaterials, vol.8, Article ID 184 (11 pages), 2018.

[12] Y.Y. Yang, M. Zhang, Z.P. Liu, K. Wang, and D.G. Yu, "Meletin sustained-release gliadin nanoparticles prepared via solvent surface modification on blending electrospraying," App. Surf. Sci., vol.434, pp.1040-1047, 2018.

[13] Y.H. WU, H.P. Li, X.X. Shi, J. Wan, Y.F. Liu, and D.G. Yu, "Effective utilization of the electrostatic repulsion for improved alignment of electrospun nanofibers," J. Nanomater., vol.2016, Article ID2067383, 2016.

[14] X.Y. Li, Z.B. Zheng, D.G. Yu, X.K. Liu, Y.L. Qu, and H.L. Li, "Electrosprayed sperical ethylcellulose nanoparticles for an improved sustained-release profile of anticancer drug," Cellulose, vol.24, pp.5551-5564, 2017.

[15] Z.P. Liu, Y.Y. Zhang, D.G. Yu, D. Wu, and H.L. Li, "Fabrication of sustained-release zein nanoparticles via modified coaxial electrospraying," Chem. Eng. J., vol.334, pp.807-816, 2018.

[16] H.F. Wen, C. Yang, D.G. Yu, X.Y. Li, and D.F. Zhang, "Electrospun zein nanoribbons for treatment of lead-contained wastewater," Chem. Eng. J., vol.290, pp.263-272, April 2016.

[17] Y.Y. Yang, Z.P. Liu, D.G. Yu, K. Wang, P. Liu, X. Chen, "Colonspecific pulsatile drug release provided by electrospun shellac nanocoating on hydrophilic amorphous composites," Int. J. Nanomed., vol.2018, DOI: 10.2147/IJN.S154849, 2018.

[18] G.Z. Yang, H.P. Li, J.H. Yang, J. Wan, and D.G. Yu, "Influence of working temperature on the formation of electrospun polymer nanofibers," Nanoscale Res. Lett., vol.12, Article 15, January 2017.

[19] M. Jin, D.G. Yu, C.F.G.C. Geraldes, G.R. Williams, and S.W.A. Bligh, "Theranostic fibers for simultaneous imaging and drug delivery," Mol. Pharm., vol.13, pp.2457-2465, July 2016.

[20] M. Jin, D.G. Yu, X. Wang, C.F.G.C. Geraldes, G.R. Williams, and S.W.A. Bligh, "Electrospun contrast agent-loaded fibers for colontargeted MRI," Adv. Healthcare Mater., vol.5, pp.977-985, April 2016.

[21] D.G. Yu, J.J. Li, M. Zhang, and G.R. Williams, "High-quality Janus nanofibers prepared using three-fluid electrospinning," Chem. Commun., vol.53, pp.4542-4545, April 2017.

[22] G.Z. Yang, J.J. Li, D.G. Yu, M.F. He, J.H. Yang, and G.R. Williams, "Nanosized sustained-release drug depots fabricated using modified triaxial electrospinning," Acta Biomater., vol.53, pp.233-241, April 2017.

[23] Y.H. WU, C. Yang, X.Y. Li, J.Y. Zhu, and D.G. Yu, "Medicated nanofibers fabricated using $\mathrm{NaCl}$ solutions as shell fluids in a modified coaxial electrospinning," J. Nanomater., vol.2016, Article ID 8970213. 2016.

[24] K. Wang, H.F. Wen, D.G. Yu, Y. Yang, and D.F. Zhang, "Electrosprayed hydrophilic nanocomposites coated with shellac for colon-specific delayed drug delivery," Mater. Design, vol.143, pp.248255, 2018.

[25] Z.P. Liu, L.L. Zhang, Y.Y. Yang, D. Wu,G. Jiang, and D.G. Yu, "Preparing composite nanoparticles for immediate drug release by modifying electrohydrodynamic interfaces during electrospraying," Powd. Technol., vol.327, pp.179-187, 2018.

[26] B. Sanchez-Vazquez, A.J. Amaral, D.G. Yu, G. Pasparakis, and G.R. Williams, "Electrosprayed Janus particles for combined photochemotherapy," AAPS PharmSciTech, vol.8, pp.1460-1468, 2017.

[27] Q. Wang, H.P. Li, C. Yang, J.J. Li, and D.G. Yu, "Beads-on-a-string amorphous solid dispersion fabricated using a modified coaxial electrospinning,” J. Control. Release, vol.259, pp.e111-e112, 2017.

[28] Y. Xu, J.J. Li, D.G. Yu, G.R. Williams, J.H. Yang, and X. Wang, "Influence of the drug distribution in electrospun gliadin fibers on drugrelease behavior," Eur. J. Pharm. Sci., vol.106, pp.422-430, August 2017.

[29] A. Gusarov, and I. Smurov, "Gas-dynamic boundary conditions of evaporation and condensation: Numerical analysis of the Knudsen layer," Phys. Fluids, vol.14, pp. 4242-455, 2002. 